\title{
KRIGING METAMODELING IN MULTI-OBJECTIVE SIMULATION OPTIMIZATION
}

\author{
Mehdi Zakerifar \\ William E. Biles \\ Gerald W. Evans \\ Dept. of Industrial Engineering \\ University of Louisville \\ Louisville, KY, 40292, USA
}

\begin{abstract}
This paper describes an experiment exploring the potential of kriging metamodeling for multi-objective simulation optimization. The experiment studies an $(s, S)$ inventory system with the objective of finding the optimal values of reorder point $s$ and maximum inventory level $S$ so as to minimize the total cost of the system while maximizing customer satisfaction. This experiment compares classical response surface methodology to kriging metamodeling as experimental approaches. The results of this experiment indicate that kriging metamodeling offers new opportunities for solving multi-objective optimization problems in stochastic simulation.
\end{abstract}

\section{INTRODUCTION}

A metamodel, also called a response surface, is an approximation of an input/output (I/O) function that is defined by an underlying simulation model (see Kleijnen, 2008). The metamodel is the surrogate for the real-world system that is used for experimentation and analysis; that is, experimentation with the actual system is far too costly and time consuming, so that computer-based experimentation, or simulation, is preferred. Most metamodeling studies focus on low-order polynomial regression using factorial-based experimental designs. For the first-order regression model with $n$ design variables $x_{i}, i=$ $1, \ldots n$, the form of the regression metamodel is

$$
\mathrm{E}(\mathrm{y})=\beta_{0}+\beta_{1} \mathrm{x}_{1}+\ldots+\beta_{\mathrm{n}} \mathrm{x}_{\mathrm{n}}
$$

while the second-order model is given by

$$
\mathrm{E}(\mathrm{y})=\beta_{0}+\sum_{\mathrm{i}} \beta_{\mathrm{i}} \mathrm{x}_{\mathrm{i}}+\sum_{\mathrm{i}} \beta_{\mathrm{ii}} \mathrm{x}_{\mathrm{i}}^{2}+\sum_{\mathrm{i}} \sum_{\mathrm{j}} \beta_{\mathrm{ij}} \mathrm{x}_{\mathrm{i}} \mathrm{x}_{\mathrm{j}}
$$

The classic design used to estimate a first-order metamodel is the factorial design, or in the case where $n$ is large (say, $n>5$ ), the fractional factorial design. For the second-order metamodel, the central-composite design is the experimental design most often used. Kleijnen (2008) gives an excellent treatise on the development of regression metamodels in simulation.

\section{MULTI-OBJECTIVE OPTIMIZATION}

The multiple objective optimization problem can be written as follows:

$$
\begin{gathered}
\max (\min ) G\left(f_{j}(\mathbf{x}), j=1, \ldots, m\right) \\
\text { subject to } \mathbf{a} \leq \mathbf{x} \leq \mathbf{b}
\end{gathered}
$$

where $\mathbf{x}$ is the $n$-vector of input factors $\mathrm{x}_{\mathrm{i}}, \mathrm{i}=1, \ldots, \mathrm{n}, \mathbf{a}$ and $\mathbf{b}$ are lower bounds and upper bounds, respectively, on $\mathbf{x}$, and the function $\mathrm{G}(\mathrm{)})$ is a policy for prioritizing the $\mathrm{f}_{\mathrm{j}}(\mathbf{x}), \mathrm{j}=1, \ldots, \mathrm{m}$. 
Hawe and Sykulski (2008) discuss several algorithms by which to apply kriging to the solution of the so-called MOOP (Multiple Objective Optimization Problem) shown in (3) above, but the one evaluated here is the approach of transforming

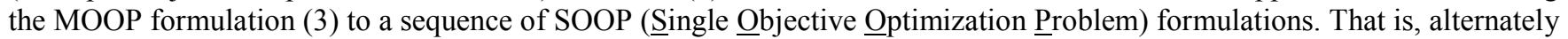
solve the constrained optimization problem (4) below until an optimal solution is found:

$$
\begin{gathered}
\qquad \max (\min ) \mathrm{f}_{\mathrm{j}}(\mathbf{x}) \text { for some } j \\
\text { subject to constraints } \mathrm{f}_{\mathrm{k}}(\mathbf{x})\{\leq,=, \geq\} \mathbf{c}, \mathrm{k}=1, \ldots, \mathrm{m} \text { for } \mathrm{k} \neq \mathrm{j} \text { and } \mathbf{a} \leq \mathbf{x} \leq \mathbf{b}
\end{gathered}
$$

where $\mathbf{c}$ is the vector of bounds on the objective functions $f_{k}(\mathbf{x}), k=1, \ldots, m$.

Another approach to solving the MOOP problem is to weight each of the $m$ objective functions $f_{j}(\mathbf{x}), j=1, \ldots, m$ with a weight $w_{j}, j=1, \ldots m$ to form the weighted objective $\sum w_{j} f(x)$ such that $0 \leq w_{j} \leq 1$ and $\Sigma w_{j}=1$. For an objective $f_{j}(\mathbf{x})$ which is to be maximized, the coefficient of $\mathrm{w}_{\mathrm{j}}$ is 1 , whereas for those objectives being minimized the coefficient of $\mathrm{w}_{\mathrm{j}}$ is -1 . The con-

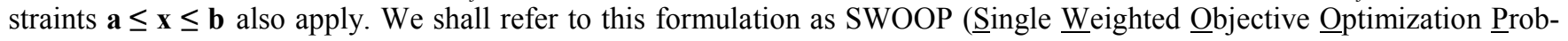
lem).

\section{RESPONSE SURFACE METHODOLOGY (RSM)}

As stated earlier, most metamodeling studies focus on low-order polynomial regression using factorial-based experimental designs. The central composite design (CCD) is a popular experimental design for estimating a quadratic regression in a local, unimodal region of the design space. Figure 1 illustrates a spreadsheet showing the deployment of $k=2^{n}+2 n+c$ design points, where $n$ is the number of input parameters or design variables, and $c$ is the number of replications of the center point of the design space which is selected to achieve some desired statistical performance in the regression models. In the case shown in Figure $1, n=2$ and $c=5$, therefore $k=13$. There are $2^{2}=4$ points arrayed in a square (generally, an $n$-dimensional hypercube with $2^{n}$ corner points), $2 x 2=4$ points along the two axes (generally, $2 n$ ), and $c=5$ replications of the center point. In the case of simulation experimentation, $r$ replications of the simulation are carried out at each of the design points using common random numbers, except in the case of the $c$ repetitions of the center point where independent random number streams must be employed. The mean values for each of the $m$ simulation responses are recorded and multiple regression is applied to fit the response functions $\boldsymbol{y}_{j}(\boldsymbol{x}), j=1, \ldots, m$. An optimization procedure (e.g., LINDO, 2008) is used to find an optimal solution to the problem formulation of (3) or (4) above.



Figure 1: A Central Composite Design for the $(\mathrm{s}, \mathrm{S})$ Inventory System 
To illustrate the regression metamodeling procedure using the central composite design (CCD) approach, an Arena model of the $(s, S)$ inventory system was simulated (Kelton et al, 2007). The multi-objective optimization formulation of this system is to find the values of maximum inventory position $S$, or $x_{1}$, and the reorder point $s$, or $x_{2}$, so as to minimize total cost $y_{0}(\boldsymbol{x})$, while maximizing customer satisfaction $y_{1}(\boldsymbol{x})$, subject to the non-negativity conditions $x_{1} \geq 0$ and $x_{2} \geq 0$ and the constraint $x_{1}>x_{2}($ or $S>s)$. Total Cost is the sum of holding cost, ordering cost and shortage cost, while customer satisfaction is the proportion of customers whose demand is met immediately without resorting to backorders. The Arena-based $(S, S)$ model was simulated for $r=5$ replications at each of the $k=13$ design points in the CCD, yielding the results shown in Table1. The response surfaces for total cost $y_{0}(\boldsymbol{x})$ and $y_{1}(\boldsymbol{x})$ are shown in Figure 2. It should be pointed out that the Minitab (2007) surface graph function utilizes the raw data and does not necessarily produce very regular looking surface graphs.

The quadratic regression models for total cost and customer satisfaction are, respectively,

$$
\begin{gathered}
y_{0}=145-0.310 \mathrm{~s}-0.507 \mathrm{~S}+0.00928 \mathrm{~s}^{2}+0.00439 \mathrm{~S}^{2}-0.00318 \mathrm{~s} * \mathrm{~S} \\
y_{l}=-0.154+0.0270 \mathrm{~s}+0.0159 \mathrm{~S}-0.000188 \mathrm{~s}^{2}-0.000064 \mathrm{~S}^{2}-0.00015 \mathrm{~s} * \mathrm{~S}
\end{gathered}
$$

First solving the constrained optimization problem to minimize total cost $y_{0}$ subject to the constraint that customer satisfaction $y_{1} \geq 0.95$, we find the solution $y_{0}=\$ 123.36$ and $y_{1}=0.952$ at $s=28$ and $S=68$. Then solving the alternate problem of maximizing customer satisfaction $y_{1}$ subject to the constraint that total cost $\leq \$ 124$, we obtain the solution $y_{0}=\$ 124$ and $y_{l}=1$ at $s=36$ and $S=76$. No better solution was found.

Table 1: Simulation Results for a CCD Experimental Design with the (s, S) Inventory System

\begin{tabular}{|c|c|c|c|c|c|c|c|}
\hline & $\mathbf{S}$ & $\mathbf{S}$ & $\mathbf{s}^{\wedge}$ & $\mathbf{\mathbf { S } ^ { \wedge } \mathbf { 2 }}$ & $\mathbf{s} * \mathbf{S}$ & $\begin{array}{c}\text { Total Cost } \\
\mathbf{\$}\end{array}$ & $\begin{array}{c}\text { Customer } \\
\text { Satisfaction }\end{array}$ \\
\hline 1 & 24 & 75 & 576 & 5625 & 1800 & 119.78 & 0.944 \\
\hline 2 & 34 & 50 & 1156 & 2500 & 1700 & 125.24 & 0.948 \\
\hline 3 & 34 & 100 & 1156 & 10000 & 3400 & 128.74 & 0.992 \\
\hline 4 & 14 & 50 & 196 & 2500 & 700 & 123.66 & 0.725 \\
\hline 5 & 14 & 100 & 196 & 10000 & 1400 & 130.34 & 0.921 \\
\hline 6 & 24 & 40 & 576 & 1600 & 960 & 128.18 & 0.755 \\
\hline 7 & 24 & 110 & 576 & 12100 & 2640 & 131.55 & 0.969 \\
\hline 8 & 10 & 75 & 100 & 5625 & 750 & 129.23 & 0.820 \\
\hline 9 & 38 & 75 & 1444 & 5625 & 2850 & 123.37 & 0.986 \\
\hline 10 & 24 & 75 & 576 & 5625 & 1800 & 124.61 & 0.953 \\
\hline 11 & 24 & 75 & 576 & 5625 & 1800 & 126.54 & 0.935 \\
\hline 12 & 24 & 75 & 576 & 5625 & 1800 & 124.48 & 0.952 \\
\hline 13 & 24 & 75 & 576 & 5625 & 1800 & 124.01 & 0.955 \\
\hline
\end{tabular}

\section{KRIGING METAMODELING}

Kriging is an interpolation method that predicts unknown values of a random function; see, for example, Cressie (1993) and Wackernagel (2003). More precisely, a Kriging prediction is a weighted linear combination of all output values already observed:

$$
\begin{gathered}
\hat{Y}\left(\mathbf{x}_{0}\right)=\sum_{i=1}^{n} \lambda_{i} \cdot Y\left(\mathbf{x}_{i}\right)=\lambda^{\prime} \cdot \mathbf{Y} \\
\text { with } \sum_{i=1}^{n} \lambda_{i}=1
\end{gathered}
$$




\section{Zakerifar, Biles and Evans}

The weights $\lambda$ in (5) are inversely proportional to the Euclidean distance from the prediction point $\mathbf{x}_{0}$ and the experimental points $\mathbf{x}_{\mathbf{j}}$. An extensive description of the mathematical development for the kriging metamodeling approach can be found in Biles et al (2007).



Figure 2: Surface Plots for Total Cost and Customer Satisfaction for the (s, S) Inventory System

Figure 3 is a spreadsheet representation of a Latin Hypercube Design (LHD) for an experiment involving 20 design points for the two input factors $s$ and $S$. The recommended number of design points in the LHD is $10 n$, where $n$ is the number of input factors. The column of 1's at the right side of the spreadsheet and the row of 1's at its bottom demonstrate the essential space-filling character of the LHD - that is, there must be exactly one 1 in every row and exactly one 1 in every column of the LHD. Harking back to Figure1, we see that the central composite design does not possess this feature and hence cannot be applied with kriging metamodeling.

Table 2 gives the simulation results for the 20 design points in the LHD. Figure 4 shows surface plots of total cost and customer satisfaction, respectively. The kriging features of the Matlab DACE toolbox (Lophaven et al, 2002) are applied to the data $\boldsymbol{x}$ and $\boldsymbol{y}$. The optimal solution $(\boldsymbol{x})$ * is found by examining predictions at all possible combinations of $\boldsymbol{x}$ over the experimental region and signifying a constraint violation with a 1 and non-violation with a 0 . This Boolean vector is then scanned to find the minimum total cost, or maximum customer satisfaction, among feasible kriging predictions.

The quadratic regressions for total cost $y_{0}$ and customer satisfaction $y_{l}$ are, respectively,

$$
\begin{gathered}
y_{0}=203-1.97 \mathrm{~s}-1.84 \mathrm{~S}+0.032 \mathrm{~s}^{2}+0.0134 \mathrm{~S}^{2}-0.00577 \mathrm{~s} * \mathrm{~S} \\
y_{1}=-0.475+0.0409 \mathrm{~s}+0.0204 \mathrm{~S}-0.000335 \mathrm{~s}^{2}-0.000085 \mathrm{~S}^{2}-0.000233 \mathrm{~s} * \mathrm{~S}
\end{gathered}
$$

Again the irregularity of the surfaces in Figure 4 arises because the plots use the raw data, not the estimating response function.

The results of the kriging study of the (s, S) inventory system are depicted in the Pareto chart shown in Figure 5. The red curve connecting a series of red squares shows the solution set that minimizes total cost. The green curve connecting a series of green triangles gives the solution for the maximization of customer satisfaction. The blue curve connecting a series of blue circles gives a weighted compromise between total cost and customer satisfaction. The blue X's on this Pareto chart show a set of kriging predictions for both total cost and customer satisfaction that satisfy the constraints that total cost be not more than $\$ 125$ and customer satisfaction exceed 0.96. Clearly the decision maker can choose how to meet his (her) objectives from this set of kriging predictions. For instance, there is a set of predictions that give solutions between \$122 and \$123 with customer satisfaction exceeding 0.98. Alternatively, there are predicted solutions for which total cost is less than $\$ 120$ but with customer satisfaction around 0.96 . When the two constraints are tightened, the set of orange circles show solutions for which total cost is between $\$ 120$ and $\$ 121$ with customer satisfaction exceeding 0.98 . This Pareto optimal approach is ideal for examining kriging results. 


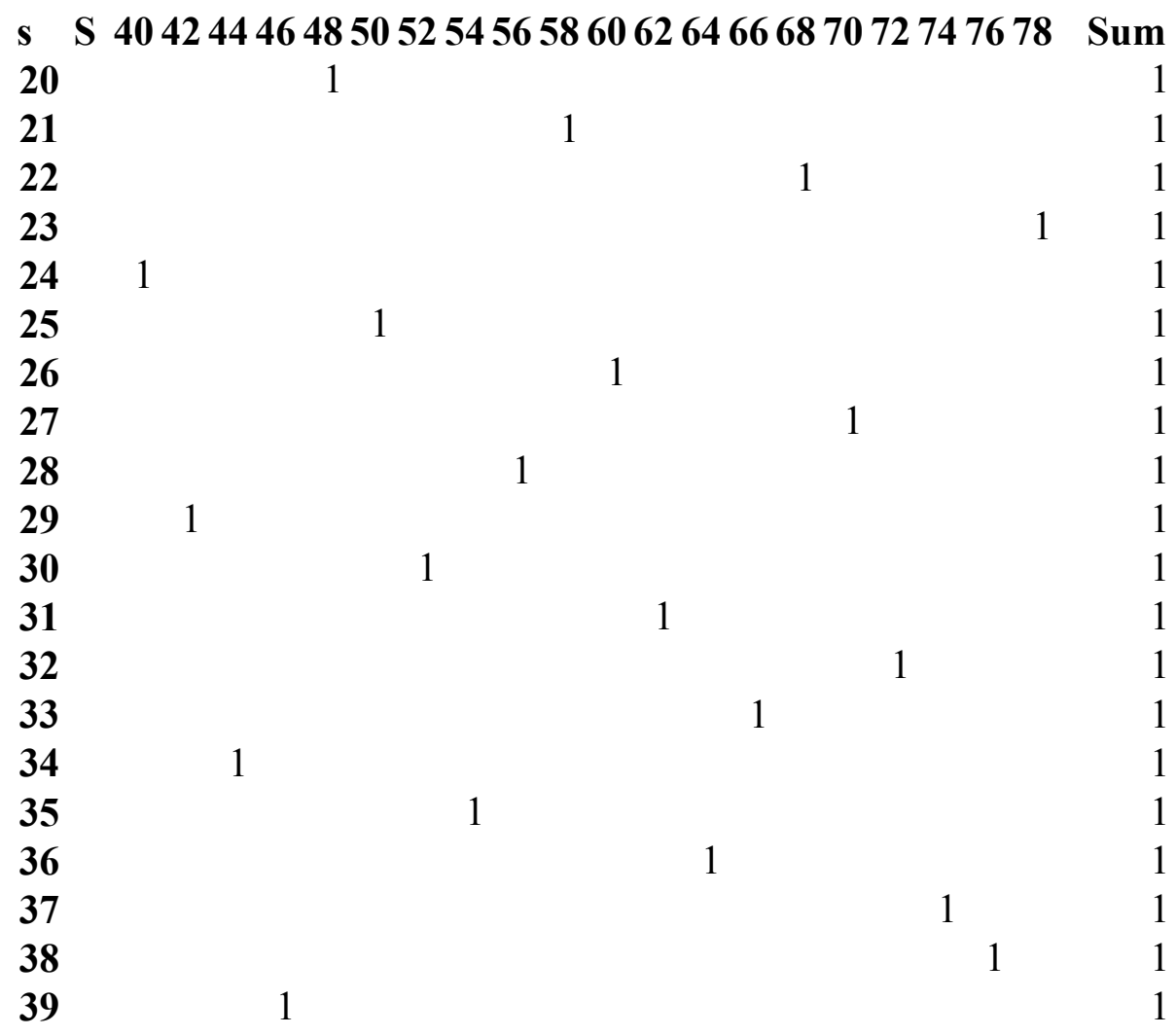

$\begin{array}{lllllllllllllllllllllll}\text { Sum } & 1 & 1 & 1 & 1 & 1 & 1 & 1 & 1 & 1 & 1 & 1 & 1 & 1 & 1 & 1 & 1 & 1 & 1 & 1 & 1 & & 20\end{array}$

Figure 3: A Latin Hypercube Design Applied to the (s, S) Inventory System

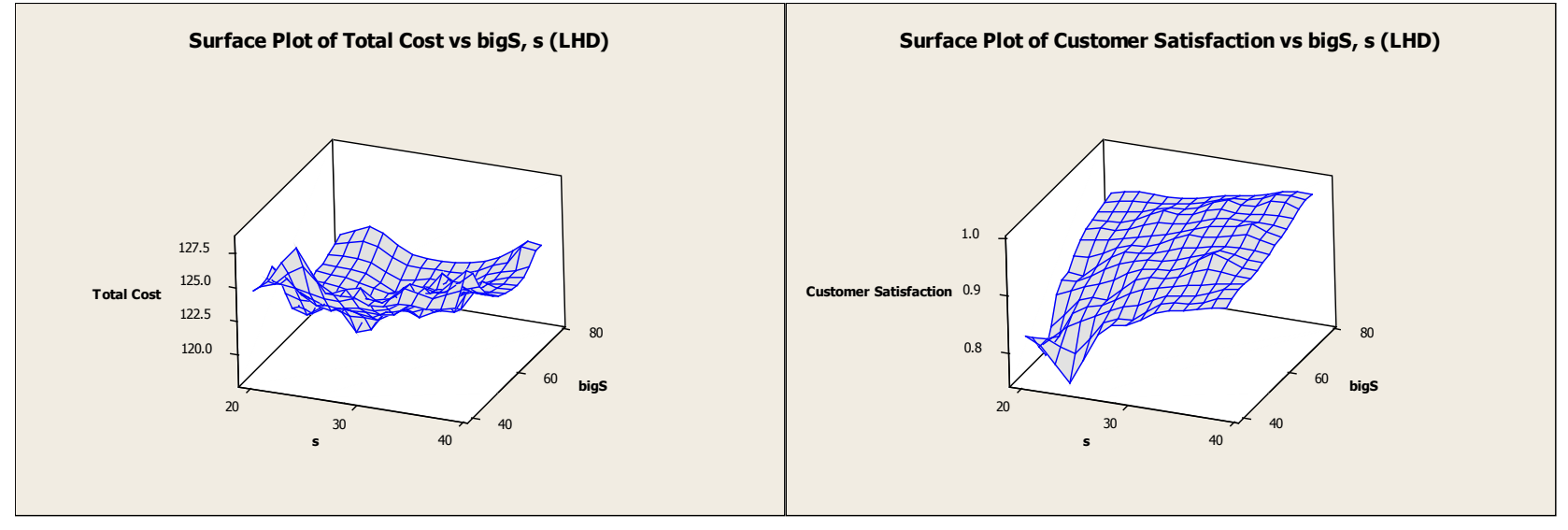

Figure 4: Surface Plots of Kriging Predictions for Total Cost and Customer Satisfaction Based on a LHD 
Zakerifar, Biles and Evans

Table 2: Simulation Results for a LHD Design with 20 Design Points

\begin{tabular}{|c|c|c|c|c|c|c|c|}
\hline & $\mathbf{s}$ & $\mathbf{S}$ & $\mathbf{s}^{\wedge} \mathbf{2}$ & $\mathbf{S}^{\wedge 2}$ & $\mathbf{s} * \mathbf{S}$ & $\begin{array}{c}\text { Total Cost } \\
\mathbf{S}\end{array}$ & $\begin{array}{c}\text { Customer } \\
\text { Satisfaction }\end{array}$ \\
\hline 1 & 20 & 48 & 400 & 2304 & 960 & 124.99 & 0.759 \\
\hline 2 & 21 & 58 & 441 & 3364 & 1218 & 119.63 & 0.855 \\
\hline 3 & 22 & 68 & 484 & 4624 & 1496 & 121.41 & 0.911 \\
\hline 4 & 23 & 78 & 529 & 6084 & 1794 & 123.27 & 0.936 \\
\hline 5 & 24 & 40 & 576 & 1600 & 960 & 128.18 & 0.755 \\
\hline 6 & 25 & 50 & 625 & 2500 & 1250 & 122.06 & 0.856 \\
\hline 7 & 26 & 60 & 676 & 3600 & 1560 & 118.24 & 0.915 \\
\hline 8 & 27 & 70 & 729 & 4900 & 1890 & 120.27 & 0.947 \\
\hline 9 & 28 & 56 & 784 & 3136 & 1568 & 120.62 & 0.918 \\
\hline 10 & 29 & 42 & 841 & 1764 & 1218 & 125.62 & 0.866 \\
\hline 11 & 30 & 52 & 900 & 2704 & 1560 & 121.96 & 0.916 \\
\hline 12 & 31 & 62 & 961 & 3844 & 1922 & 120.23 & 0.949 \\
\hline 13 & 32 & 72 & 1024 & 5184 & 2304 & 121.08 & 0.972 \\
\hline 14 & 33 & 66 & 1089 & 4356 & 2178 & 119.68 & 0.966 \\
\hline 15 & 34 & 44 & 1156 & 1936 & 1496 & 126.22 & 0.913 \\
\hline 16 & 35 & 54 & 1225 & 2916 & 1890 & 125.45 & 0.961 \\
\hline 17 & 36 & 64 & 1296 & 4096 & 2304 & 123.20 & 0.973 \\
\hline 18 & 37 & 74 & 1369 & 5476 & 2738 & 122.26 & 0.986 \\
\hline 19 & 38 & 76 & 1444 & 5776 & 2888 & 124.74 & 0.985 \\
\hline 20 & 39 & 46 & 1521 & 2116 & 1794 & 127.47 & 0.942 \\
\hline
\end{tabular}

\section{CONCLUSIONS}

This experiment has shown that kriging metamodeling has the potential to identify superior solutions to those obtained by classical response surface approaches when faced with a multi-objective optimization environment. This superiority traces both to the space-filling character of Latin Hypercube Designs, as opposed to the relative sparceness of design points in a central composite design, as well as to the more accurate predictions offered by the kriging approach. One downside to kriging metamodeling, however, is that it only works when there are a relatively large number of design points in the LHD. We tried without success an LHD of 10 design points to a simulation model of a cellular manufacturing system having 4 input factors. The four input factors were the queue capacities at each of four machine cells and were restricted to integer values. Yet, the finite queues would ideally only range in size from 1 to 5 (the mean queue sizes for typical simulation trials were in the range from 1.5 to 3 ), so even letting $1 \leq x_{i} \leq 10$ was a stretch. In this case, CCD designs permitted $1 \leq x_{i} \leq 5$ and gave excellent results. An LHD design over the same range would have only had 5 design points, far fewer than the $10 n$ recommended for kriging metamodeling.

We shall investigate other formulations of the multi-objective optimization problem in future investigations. The SOOP formulation studied here captures the advantages of the constrained optimization problem formulation reported by Biles et al (2007). We are able to report the highly successful application of the Pareto-based trade-off approach illustrated in Figure 5, and will pursue that avenue further in future research. 


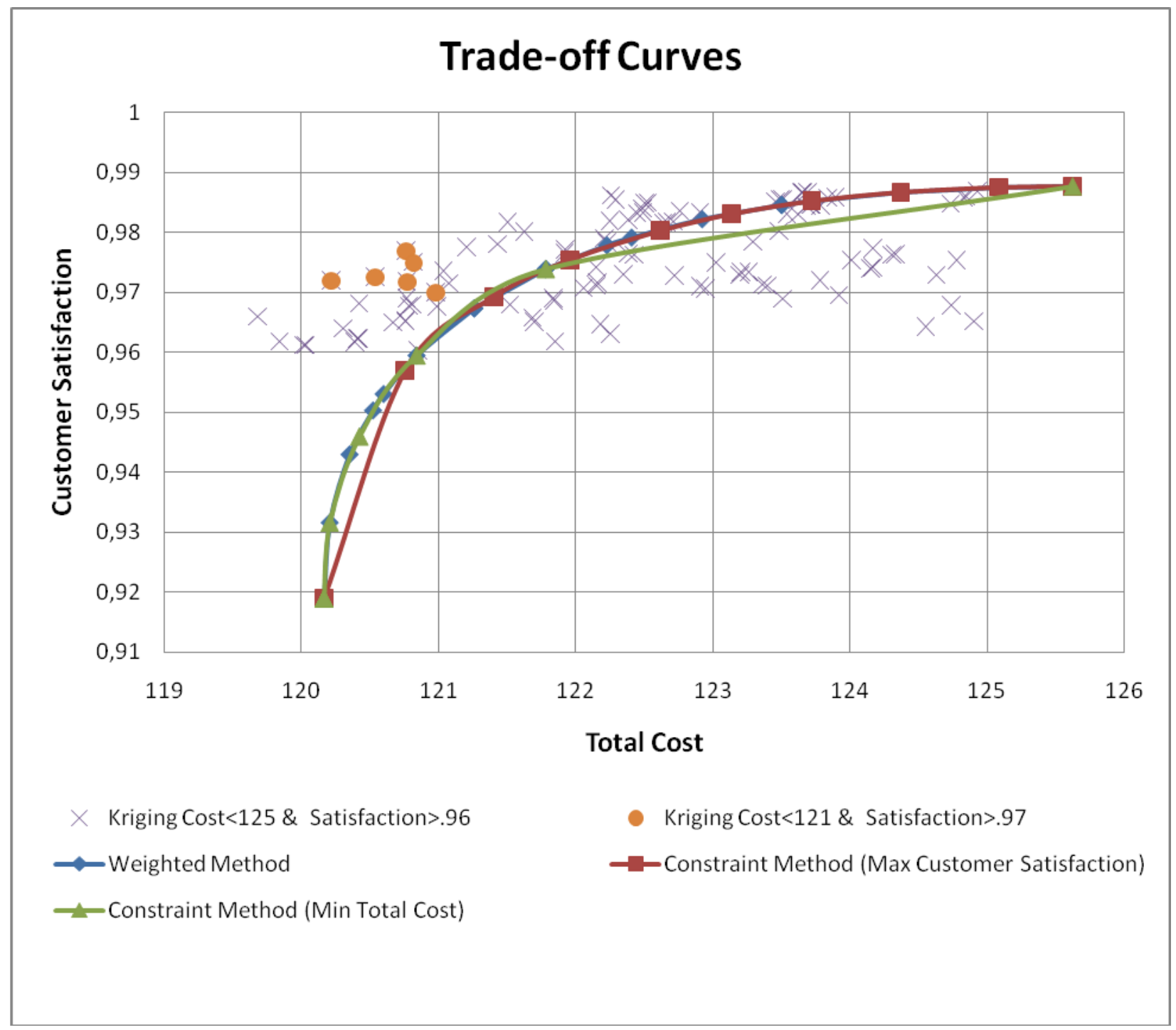

Figure 5: A Pareto Chart of Kriging Results for the (s, S) Inventory System

\section{REFERENCES}

Biles, W. E., J. P. C. Kleijnen, W. C. M. van Beers, and I. Van Nieuwenhuyse. 2007. Kriging Metamodeling in Constrained Simulation Optimization: An Explorative Study. In Proceedings of the 2007 Winter Simulation Conference, ed. S. G. Henderson, B. Biller, M-H Hsieh, J. Shortle, J. D. Tew and R. R. Barton, 362-370.

Cressie, N.A.C. 1993. Statistics for spatial data. New York: John Wiley \& Sons, Inc.

Hawe, G. and J. Sykulski. 2008. Scalarizing Cost-Effective Multi-objective Optimization Algorithms Made Possible With Kriging. In COMPEL:The International Journal for Computation and Mathematics in Electrical and Electronics Engineering, Vol. 27, No. 4: 836-844.

Kelton, W.D., R.P. Sadowski and D.T. Sturrock. 2007. Simulation with Arena. 4th ed. New York: McGraw-Hill.

Kleijnen, J.P.C. 2008. Design and Analysis of Simulation Experiments. New York: Springer.

Kleijnen, J.P.C., and W.C.M. van Beers. 2004. Application-Driven Sequential Designs for Simulation Experiments: Kriging Metamodeling. In Journal of the European Operational Research. Vol. 55, No. 9: 876-883.

Law, A.M., and W.D. Kelton. 2007. Simulation Modeling and Analysis. 4th ed. Boston, MA: McGraw-Hill.

LINDO System Inc. 2008. LINGO/Win32 11.0.0.11. Chicago, IL. 
Lophaven, S.N., H.B. Nielsen and J. Sondergaard. 2002. DACE: A Matlab Kriging Toolbox. Technical Report IMM-TR2002-12.

Matheron, G. 1963. Principles of Geostatistics. In Economic Geology, Vol. 58, No. 8: 1246-1266.

McKay, M. D., R. J. Beckman, and W. J. Conover. 1979. A Comparison of Three Methods for Selecting Input Values of Input Values in The Analysis of Output from a Computer Code. In Technometrics, 21, No. 2: 239-245.

Minitab, Inc. 2007. Minitab 15 Statistical Software. State College, PA.

The Math Works, Inc. 2001. MATLAB: The Language of Technical Computing.

Van Beers, W.C.M., and J.P.C. Kleijnen. 2003. Kriging for Interpolation in Random Simulation. In Journal of the Operational Research Society, No. 54: 255-262.

Wackernagel, H. 2003. Multivariate geostatistics. Berlin: Springer-Verlag.

\section{AUTHOR BIOGRAPHIES}

MEHDI ZAKERIFAR is a Ph.D. student in the Department of Industrial Engineering at the University of Louisville. He received his B.S. degree in Applied Mathematics from the University of Tehran (Tehran, Iran) in 2000, and his Master Degree in Industrial Engineering from Sharif University of Technology (Tehran, Iran) in 2003. He worked as an Industrial Engineer, Systems Analyst, and later Network Administrator, Database Manager and Software Developer. He also has worked as a Teacher Assistantship at the University of Louisville since 2007. His research interests include simulation modeling and optimization, decision analysis, multi-criteria and applied optimization. He can be reached by e-mail at: $<$ mozake01@louisville.edu>.

WILLIAM E. BILES is the Clark Chair of Computer Aided Engineering in the Department of Industrial Engineering at the University of Louisville. He spent two years in the US Army and seven years in industrial R\&D at Union Carbide's Advanced Materials Laboratory and at Thiokol Chemical Corporation before undertaking an academic career. He has held academic appointments at the University of Notre Dame, Penn State, Louisiana State University, and the University of Louisville. Dr. Biles received the Distinguished Engineering Alumnus award from the University of Alabama in Huntsville in 2004. His most recent publication is "Environmentally Benign Manufacturing," Chapter 1 in the 2007 John Wiley handbook Environmentally Conscious Manufacturing. His email address is <webile0lelouisville.edu>

GERALD W. EVANS is a Professor in the Department of Industrial Engineering at the University of Louisville. He has a B.S. degree in Mathematics, and M.S. and Ph.D. degrees in Industrial Engineering, all from Purdue University. Before entering academia, he worked as an Industrial Engineer for Rock Island Arsenal, and as a Senior Research Engineer for General Motors Research Laboratories. Besides simulation modeling and analysis, his research interests include multi-objective optimization, decision analysis, and discrete optimization. $\mathrm{He}$ can be reached by e-mail at <gwevan01@louisville.edu>. 